\title{
Répartition des glossines au Cameroun français (1953) *
}

\author{
par J. RAGEAU et J.-P. ADAM
}

Nous avons établi une carte provisoire qui donne la répartition géographique des onze espèces et deux variétés de glossines actuellement connues au Cameroun français. Ce sont :

Glossina palpalis Robineau-Desvoidy, 1830.

G. fuscipes Newstead, 1910 .

G. caliginea Austen, 1911.

G. pallicera Bigot, 1891 .

G. newsteadi Austen, 1929.

G. tachinoildes Westwood, 1850.

G. morsitans Westwood, 1850, var. submorsitans, Newstead, 1910.

G. longipalpis Wiedemann, 1830.

G. fusca fusca Walker, 1849.

$G$. fusca congolensis Newstead et Evans, 1921.

G. fuscipleuris Austen, 1909.

G. haningtoni Newstead et Evans, 1922.

G. tabaniformis Westwood, 1850.

Une douzième espèce, $G$. nigrofusca Newstead, 1910, a été trouvée par F. Zumpt (1936) au Cameroun britannique : plaine de Tiko, Mamfe (Ossidinge), Elephantensee; son existence est donc possible dans les régions de Moungo et Bamiléké, limitrophes du territoire sous mandat britannique.

L'étude chorologique des tsé-tsé fut entreprise au Cameroun dès le début du siècle par les entomologistes et médecins allemands (Zupilza, 1908; Gruenberg, 1912) et Glaeser (1914) fit une première mise au point à ce sujet. Zumpt (1936) reprit ces recherches au Cameroun britannique et Guibert (1937) au Cameroun français. Plus récemment Vaucel (1943) et Beaudiment (1948) résumèrent nos connaissances sur la distribution des glossines au Territoire.

C'est sur ces travaux et aussi sur les registres d'identification des glossines tenues au Laboratoire d'Entomologie du Service de Santé, puis du Service d'Hygiène Mobile et de Prophylaxie de Yaoundé depuis 1937, que nous nous sommes basés pour établir la présente carte.

Nous avons pu vérifier un certain nombre des points de capture mentionnés dans ces registres et en ajouter de nouveaux, soit au cours de nos multiples toumées de 1948 à 1953, soit en déterminant les spécimens de glossines envoyés : par différents postes médicaux du territoire : Douala, Kribi, Edéa, Bafia, Abony-Mbang, Doumé, Bertoua, Batouri, Garoua et Fort-Foureau. Pour les espèces rares, $G$. haningtoni par exemple, nous avons relevé des provenances nouvelles qui étendent leur répartition.

Cependant, malgré la multiplicité des localisations que nous avons notées, il reste des incertitudes sur la limite nord de la zone à $G$. palpalis, la séparation entre le domaine de $G$. palpalis s. str. et celui de G. fuscipes, la distribution des espèces dans les régions de l'Adamaoua et du Nord-Cameroun. Seules les prospections détaillées en saison sèche et en saison des pluies, notamment dans les contrées situées au nord d'une ligne Banyo-Bétaré Oya, nous permettront de compléter cette carte et de délimiter avec précision les aires d'extension des différentes glossines.

$$
*^{*}{ }^{*}
$$

Deux espèces couvrent des zones très étendues et présentent une particulière importance au point de vue médico-vétérinaire : $G$. palpalis s. l. dans le Sud-Cameroun, $G$. tachinoïdes dans le nord. Les autres ont des peuplements soit localisés (comme $G$. caliginea), soit de faible densité (comme $G$. fusca) et leur rôle pathogène paraît moindre.

La distribution des tsé-tsé, presque,uniforme dans toute la zone forestière, devient discontinue en zone de savane pour se cantonner aux abords du réseau hydrographique dans les régions sahéliennes de la Bénoué et du Logone-Chari. Les glossines disparaissent également dans les contrées montagneuses déboisées d'altitude supérieure à 1.200 m : monts Manengouba, plateau Bamiléké (Dschang), plateau de Koutaba, monts Yakounga, Bamboutos, hauteurs de l'Adamaoua, monts Alantika, Kapsiki...

* Travail présenté à la Société de Pathologie Exotique lors de la séance du 14 Octobre 1953. 


\section{$*^{*} *$}

Pour la représentation graphique des espèces camerounaises, nous avons suivi les directives de la Conférence Interafricaine de la Tsé-tsé et de la Trypanosomiase (Brazzaville, Février 1948) en ut1lisant les couleurs et symboles internationaux. Les symboles ont été employés principalement pour les espèces localisées, les coloris uniquement pour les 3 espèces couvrant de larges zones : G. palpalis, $G$. tachinoïdes et $G$. morsitans. Une première carte en couleurs au 1/3.000.000 a été adressée dès Janvier 1949 à la Direction du Service d'Hygiène Mobile et de Prophylaxie du Cameroun et au Dr Henrard, chargé de la mise à jour et de la publication de la carte des glossines pour l'Ouest africain.

Mais les difficultés de reproduction des couleurs nous ont amenés à redessiner une carte en noir et blanc, en utilisant les conventions suivantes : hachures obliques aulieu de marron pour la zone à G. palpalis; hachures verticales au lieu de vert clair pour la zone à G. morsitans; hachures horizontales au lieu de bleu clair pour la zone à $G$, tachinoïdes. Cette carte, à l'échelle approximative de 1/5.000.000, a paru dans le Rapport annuel du Gouvernement français à l'Assemblée générale des Nations-Unies sur l'Administration du Cameroun pour 1950, p. 385.

La carte actuelle est la même, à quelques corrections et additions près : raccordement des zones à G. palpalis, G. morsitans et $G$, tachinoïdes avec celles de I'A.E.F.; localités nouvelles pour G. caliginea, $G$. haningtoni, G. tabaniformis et $G$. fusca; présence de G: newsteadi dans l'Est-Cameroun. L'échelle adoptée était au $1 / 2.000 .000$ et se trouve modifiée par suite des nécessités d'impression.

\section{G. palpalis Robineau-Desvoidy.}

Cette espèce est représentée au Cameroun par deux sous-espèces :

a) G. palpalis R. D. s. str. qui couvre tout le sudouest du territoire.

b) G. palpalis fuscipes Newstead, 1910 ( = G. ziemanni Gruenberg, 1912) qui existe dans toute la région du sud-est. Cette forme a été récemment élevée au rang d'espèce par de Barros Machado (Congresso de Medicina Tropical, Lisbonne; 1952).

La zone de partage entre palpalis s. str. et fuscipes semble se situer dans la région du Haut-Nyong (Messaména et Abong-Mbang) où les deux formes ccexistent, mais avec une forte prédominance de fuscipes. Dans les régions du Lom et Kadéi et de la Boumba-Ngoko on n'observe plus que fuscipes; de même, selon Vaucel, dans les savanes de l'Adamaoua.
Par contre, palpalis se rencontre seule dans les régions de Nyong et Sanaga, Sanaga-Maritime; Wouri, Kribi, Ntem, Moungo, Bamiléké et Mbam. Dans la région côtière de Douala et Bonabéri existe une petite race sombre de G. palpalis récemment différenciée par Roubaud (1952).

La limite nord de la zone à G. palpalis s. l. atteint le $7^{\text {e }}$ degré de latitude nord, mais nous n'avons pas de dunnées suffisantes pour la fixer avec précision. Les points les plus septentrionaux où a été observée cette espèce se localisent vers Banyo, Tibati et Bétaré-Oya. En altitude, on peut la rencontrer jusqu'à $1.200 \mathrm{~m}$. dans la zone forestière.

\section{$2^{\circ}$ G. caliginea Austen.}

Cette glossine abonde dans le bassin côtier du Wouri, de la Dibarnba et de la Sanaga à des altitudes de 0 à $300 \mathrm{~m}$. Elle a surtout été étudiée à Douala et Bonabéri (Roubaud et Rageau, 1950-1952) mais des exemplaires ont été capturés dans les régions de Kribi, du Nkam, du Moungo et de la Sanaga Maritime oì elle remonte au moins jusqu'à Edéa. Sa limite nord parait se situer vers Bafang

\section{$3^{\circ}$ G. pallicera Bigot.}

Nous ne possédons pour cette espèce que des points de capture isolés et, sauf à Douala. Yabassi et Kribi, il ne nous a pas été possible de vérifier les identifications. Aussi la répartition de $G$, pallicera est-elle donnée sous réserve. 'Elle comprend les régions du Wouri (Douala, Bonabéri), Moungo (Mbanga, Nkongsamba), Nkam (Yabassi), Sanaga Maritime (Edéa, Eséka), Kribi, Ilaut-Nyong (Doumé). Guibert et Vaucel l'ont signalée également dans les régions de Lom et Kadéi (Batouri) et Boumba-Ngoko (Moloundou), mais il semble qu'il s'agisse d'une confusion avec $G$. newsteadi: $G$. pallicera remonte moins au nord que G. palpalis (elle ne semble pas dépasser Bafang); elle est toujours assez rare, localisée à la zone côtière et occidentale. Nous l'avons observée en cohabitation avec $G$. palpalis s. str. et G. caliginea à Douala.

\section{$4^{\circ}$ G. newsteadi Austen, 1929.}

Très voisine morphologiquement de G. pallicera mais à répartition géographique orientale, cette espèce semble avoir été confondue avec la précédente par Cuibert (1937) et Vaucel (1943). Nous l'avons observée uniquement dans la région de Lom et Kadéi (Doumé et Batouri) mais il est probable qu'elle se retrouvera dans tout le Sud-Est Cameroun en particulier dans les régions de la Boumba-Ngoko (subdivisions de Yokadouma et de Moloundou). I. Maillot (1953) l'a en effet signalée en Oubangui dans la région de la Haute-Sangha (Nola), limitrophe de celle de la Boumba-Ngoko. Les localisations de 
G. pallicera à Doumé, Batouri et Moloundou portées sur nos cartes précédentes seraient, en réalité, des stations de $G$. newsteadi. Espèce de grande forêt, $G$. newsteadi semble n'avoir qu'une faible densité dans le Sud-Est Cameroun.

\section{G. tachinoides Westwood.}

C'est l'espèce dominante dans le Nord-Cameroun : bassins de la Bénoué et du Logone. Les cours supérieurs de la Wina, de la Bénoué et de ses affluents occidentaux jalonnent approximativement sa limite Sud (entre le $7^{\text {e }}$ et le $8^{\mathrm{e}}$ degré de latitude nord). Sa distribution est discontinue : elle n'occupe qu'une bande étroite le long du réseau hydrographique et ne monte pas à plus de $600 \mathrm{~m}$ d'altitude. Comme provenances certaines, nous avons les environs de Poli, la vallée de la Bénoué entre Ndaou et Kinada, la vallée de la Léré, une petite zone à l'est de Guidder, la vallée du Logone (Logone-Birni, Fort-Foureau, Houlouf), celle du Chari (Goulfei) et celle du Serbéouel ainsi que les environs de Ouazza (= Wasa) à environ $50 \mathrm{~km}$ au nord-est de Mora.

\section{$6^{\circ}$ G. morsitans var. submorsitans Newstead.}

Se rencontre dans les savanes boisées à climat soudanien des régions de la Bénoué et du NordCameroun du $5^{e}$ au $12^{e}$ degré de latitude nord, à des altitudes variant de 300 à $1.000 \mathrm{~m}$. Nous avons malheureusement fort peu de renseignements sur la répartition géographique de cette espèce que nous n'avons pas encore rencontrée personnellement.

Dans les Rapports du Service de Santé (1937-1945) et sur une carte inédite laissée par le Dr Guibert, nous avons relevé comme provenances : les environs de Yoko, Ngaoundéré, Poli, Garoua, Rei-Bouba (jusqu'à la frontière) et Logone-Birni. A la frontière du Tchad, Receveur (1950) a signalé $G$. morsitans à Fianga et dans toute la vallée du Logone.

\section{$7^{\circ}$ G. longipalpis Wiedemann.}

Comme pour $G$. submorsitans, les données que nous possédons sur cette espèce sont très restreintes. Sa répartition géographique paraît voisine de celle de $G$. morsitans mais très discontinue. Ia carte inédite de Guibert indique une localisation vers le 4 e degré de latitude nord (Doumé, Bertoua) une autre vers le $5^{\mathrm{e}}$ (Yoko) et une $3^{\mathrm{e}}$ vers le $7^{\mathrm{e}}$ (Ngaoundéré). Nous n'avons pas eu l'occasion de capturer nous-mêmes G. Ingipalpis ou d'en déterminer des exemplaires.

\section{$8^{\circ}$ G. fusca Walker.}

Les grandes glossines du groupe fusca se rencontrent uniquement dans la zone forestière du sud et leur limite septentrionale semble constituée par le $6^{\mathbf{e}}$ degré de latitude nord, bien qu'on en ait signalé des captures isolées jusqu'au $8^{\star}$ degré nord. Elles vivent à une altitude de 0 à $1.000 \mathrm{~m}$ et s'observent toujours en petit nombre en raison de leur faible densité et surtout de leur horaire d'activité très matinal ou vespéral (6-8 heures et 1719 heures), parfois même nocturne. Les renseignements que nous possédons sur leur chorologie sont, par suite, fragmentaires.

$G$. fusca présente deux variétés ali Cameroun.

a) G. fusca Walker, 1849 a été trouvée dans les régions de Kribi (Kribi, Bipindi), du Wouri (Douala). du Moungo (Mbanga, Nkong-samba), du Nkam (Yabassi), Bamiléké (Bafang); Bamoun (Foumban), Mbam (Bafia, Yoko), Nyong et Sariaga (Evoduula, Saa, Nanga-Eboko, Akonolinga, Ayos), Haut-Nyong (Messaména, Abong-Mbang, Doumé. Lomié), Lom et Kadéi (Batouri, Bétaré-Oya), Boumba-Ngoko (Yokadouma, Biwala, Moloundou) $e^{\ddagger}$ même Adamaoua (Ngaoundéré ?).

b) G. fusca, var. congolensis Newstead et Evans, 1921 a été signalée par Vaucel dans la région de Mbam (subdivision de Yoko) et dans, celle de Lom et Kadéi (Bétaré-Oya).

\section{c" G. haningtoni Newstead et Evans.}

Decrite au Cameroun britannique (Mamfe) en 1922, cette glossine a été retrouvée en divers points du Sud-Cameroun depuis Yabassi à l'ouest jusqu'à Yokadouma et la frontiere de l'Oubangui à l'est. Sa limite septentrionale se situe vers le $5^{\mathrm{e}}$ degré de latitude nord. Elle existe dans les régions du Nkam (Yabassi), de Nyong et Sanaga (Yaoundé, Evodoula, Mbalmayo), du Ntem (Ebolowa, Amban), du HautNyong (Doumé), de Lom et Kadei (Batouri, Touki) et de la Boumba-Ngoko (Yokadouma, Ngola, Biwala); dans ces trois dernières régions elle paraît relativement répandue.

\section{$10^{\circ}$ G. fuscipleuris Austen.}

D'après Vaucel (1943) cette glossine d'Afrique orientale est connue au Territoire par « 33 exemplaires, tous capturés dans les environs de BétaréOya (altitude : $800 \mathrm{~m}$ ) à la frontière CamerounOubangui-Chari (région de Lom et Kadei) ». Dans les archives du Laboratoire d'Entomologie de Yaoundé, nous avons également relevé une provenance de Doumé (Haut-Nyong) mais rous n'avons pu la contrôler

\section{$11^{\circ}$ G. tabaniformis Westwood.}

C'est lespèce du groupe "fusca "İ plus ccinmune au Cameroun. Sa répartition géographique est superposable en partie à celle de $G$. fusca et on observe parfois les deux espèces dans les mêmes gites mais G. tabaniformis semble cius répandue. 
Elle vit dans toute la zone forestière du Sud-Cameroun à des altitudes de 0 à $800 \mathrm{~m}$ et elle remonte jusqu'au $6^{\mathbf{e}}$ degré de latitude nord. On connait son existence dans les régions du Wouri (Douala, Bonabéri), de la Sanaga Maritime, du Moungo (Mbanga, Nkongsamba), Bamiléké (Bafang), du Nkam (Yabassi). de Kribi, de Nyong et Sanaga (Yaoundé, Evodoula, Mbalmayo, Akonolinga, Ayos, NangaEboko), du Mbam (Yoko), du Ntem (Ebolowa; Nkoemvone, Ambam), du Haut-Nyong (Messaména, Abong-Mbang, Doumé), de Lom et Kadei (Batouri) et de la Boumba-Ngoko (Yokadouma, Biwala, Moloundou).

\section{BIBLIOGRAPHIE}

BARROS MACHADO (A. de). - Congresso de Medicina Tropical, Lisbonne, 1952.

BEAUDIMENT (R.). - Les glossines au Cameroun et la prophylaxie agronomique et insecticide. Conférence africaine sur la Tsé-tsé et la trypanosomiase, Brazzaville, 2-8 février 1948, pp. 345-350; édité par la «Documentation française ». Toulouse, 1950.

CLIESER (H.). - Bestimmungsschlüssel der in Kamerun und Togo bekannten Tse-tsearten. Arch. f. Schiffs- u. Trop. Hyg. Leipzig, 1914, 18, no 16 , pp. 571-573.

GRUENBERG (K.). - Eine neue Tsetse-Fliege aus Kamerun. Sitzungsber Gesellsch. Naturforsch. Freunde. Berlin, 1912, no 4, pp. 243-248.

GUIBERT (M.). - Les glossines du Cameroun. Bull. Soc. Path. exot. 1937, 30, 4, 284-286.

HEGH (E.). - Les Tsé-tsés. Imprimerie industrielle et financière; Bruxelles, 1, 1929.

MAILLOT (L.). - Répartition géographique des glossines en Afrique équatoriale française. B.P.I.T.T. no 192/0, Léopoldville, août 1952 et Bull. Soc. Path. exot., 16, 1953, 195-197.
MALLOT (M.). - Carte de répartition géographique des glossines en A.E.F. Office de: la Recherche Scientifique Outre-Mer, 1952.

NEWSTEAD (R.) et EVANS (A.-M.). - A new Tsetsefly from the South Cameroons. Ann. Trop. Med. Parasit. 1922, 16, p. 51.

RECEVEUR (M.). -- Répartition des glossines dans le territoire du Tchad. Conférence africaine sur la Tsé-tsé et la Trypanosomiase, Brazzaville, février 1948, pp. 338-344.

ROUBAUD (E.) et RAGEAU (J.). - Glossina caliginea Austen au Cameroun français. C. R. Acad. Sci. Paris, 1950, 230, pp. 895-897.

ROUBAUD (E.), MAILLOT (L.) et RAGEAU (Ji). L'infection naturelle de Glossinea caliginea dans les gîtes à palétuviers de Douala ( $1^{\mathrm{r}} \mathrm{e}$ et $2^{\mathrm{e}}$ note). Bull. Soc. Path. exot. 11, 1951, no 5-6, pp. 309-313 et $45,1952, \mathrm{n}^{\circ} 2$, pp. 206-208.

ROUBAUD (E.), RACEAU (J.) et ADAM (J.-P.). Pupe de Glossina caliginea Aust. ibid. 45, 1922, no 1-2, pp. 10-11.

ROUBAUD (E.). - La Glossina palpalis des zones côtières à palétuviers de Douala. Ibid. $45, n^{\circ} 3$, pp. 389-395, 1952.

ROUSSEAU (L.). - Maladics parasitaires à Douala. Bull. Soc. Path. exot., 1918, 11, 8, 744-759.

VAUCEL (M.). - Glossines du Cameroun français. Rev. Sci. Méd. Pharm. Vét. Atr. tr. libre. Brazzaville, $1943,2, \mathrm{n}^{\circ} 2$, pp. 97-100.

ZUMPT (F.). - Die Tsetsefliegen, ihre Erkennungsmerkmale, Lebensweise und Bekampfung. G. Fischer, Iéna, 1936.

ZUMPT (F.). - Untersuchungen über Tsetsefliegen und deren Bekämpfung in Pflanzungsgebiet des Kamerunberges. Tropenpflanzer, Berlin, 1937, $40, n^{0} 1-2$, p. 1-31.

ZUPITZA (M.). - Ueber die Schlafkrankheitsfliege bei Duala Archiv. f. Schiffs u. Trop. Hyg. Leipzig, 1908, Beiheft, v. 12, p. 25. 


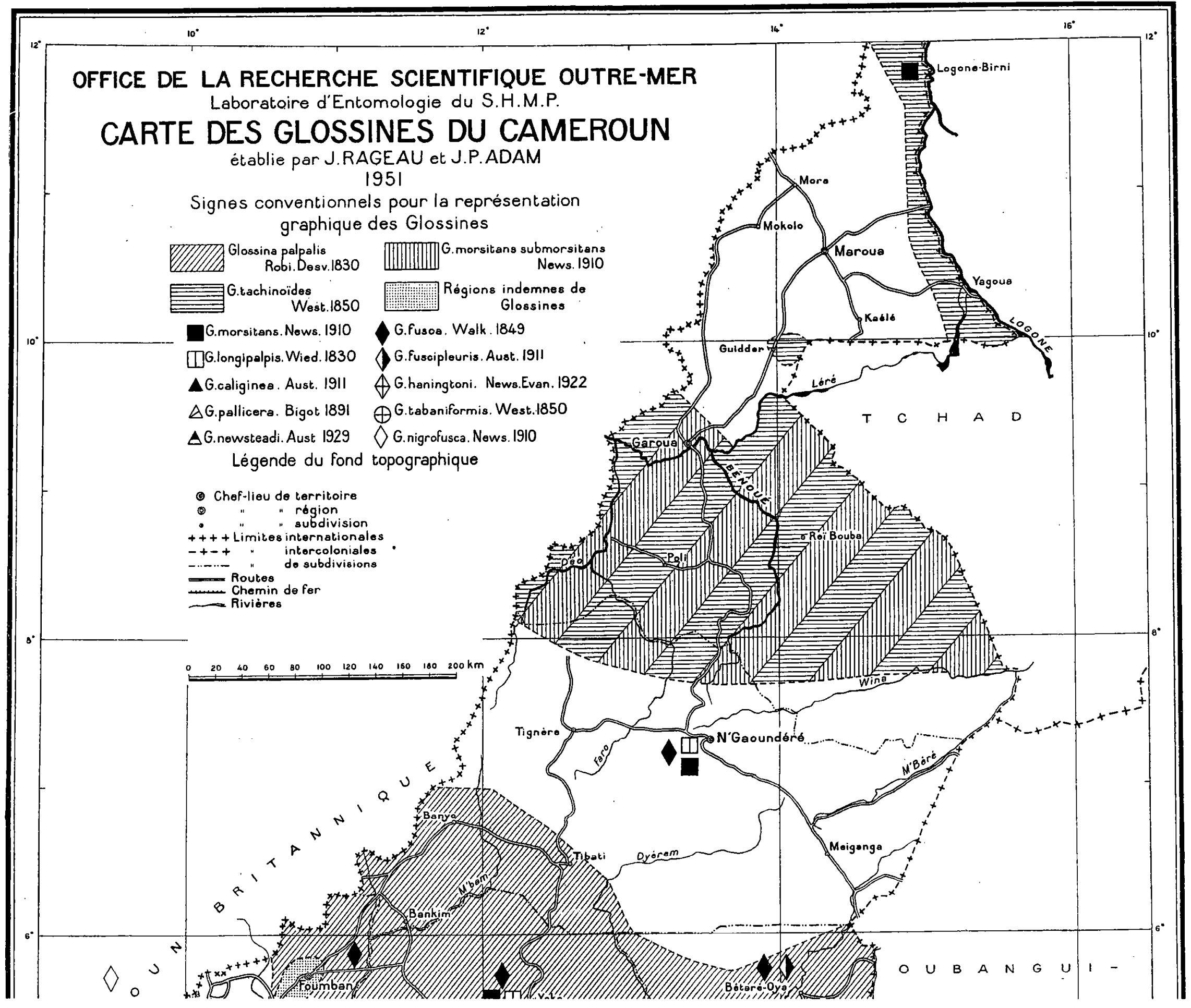


